\title{
Assessment of the effects of frequent ventricular extra systoles on the left ventricle function using two-dimensional speckle tracking echocardiography in apparently normal hearts
}

\author{
Sameh R. Allam, Mohamed Sami, Muhammad I. Azzam* \\ Department of Cardiovascular, Faculty of Medicine, Al-Azhar University, Cairo, Egypt \\ *Corresponding author: Muhammad I. Azzam; Mobile: 01004363095; Email: muhamedazzam02@gmail.com
}

\begin{abstract}
Background: premature ventricular contractions are commonly seen within the general population and frequently observed during clinical practice. In recent years studies have shown that athough PVCs was considered as mostly benign, frequent PVCs without structural heart disease might lead to cardiac dysfunction and even cardiomyopathy, more recent work has revealed that PVCs can contribute to cardiomyopathy and heart failure and treating PVCs could lead to improved cardiac function.
\end{abstract}

Aim of the Work: the aim of this work was to assess the effect of ventricular extrasystoles (VESs) on LV function by using 2D-STE for early detection of subtle LV dysfunction in patients with apparently normal hearts and assess correlation between frequency of extrasystole and degree of impairment of LV dysfunction. Patients and Methods: the ethical approval was obtained from the hospital ethical research committee and each patient entering the study was signed an informed consent. Fifty patients were included in this study with asymptomatic frequent PVCs. All were without structural heart disease. Detailed history, physical examination, full labs, resting ECG, Holter monitoring for 24 hours, conventional ECHO assessment and finally strain imaging with measurement of LV-GLS were done. They were recruited from Cardiovascular Department at Sayed Galal University Hospitals during the period from December 2017 to November 2018. In this study we assessed speckle tracking of LV in apparently normal patients with frequent PVCs.

Results: early detection of subtle LV dysfunction was done by speckle tracking echocardiographic modalities other than conventional echocardiographic and showed a significant correlation with PVCs burden, focality, interpolated PVCs and duration of the complex of ectopic beat.Conclusion: frequent PVCs may produce LV dysfunction and cardiomyopathy independently of any pre-existing underlying cardiac disease and early detection of subtle LV dysfunction can be done by speckle tracking echocardiographic modalities even in asymptomatic patient.

Keywords: echocardiography, premature ventricular contraction, interpolated PVCs, PVCs burden, speckle tracking echocardiography.

\section{INTRODUCTION}

PVCs were found in a healthy population in only $0.6 \%$ of those $<20$ years of age and $2.7 \%$ of those $>50$ years of age on 12-lead ECGs, about $50 \%$ of all people with or without heart disease in longer term monitoring ${ }^{(1)}$.Although PVCs was used considered as mostly benign, long-term frequent PVCs may lead to several cardiac complication such as cardiomegaly, cardiac hypofunction, and even PVC-induced cardiomyopathy in some patients, via pathogenic mechanisms possibly involving ventricular systole desynchrony during PVCs, shortened coupling intervals and post extra systolic potentiation ${ }^{(2)}$. Another work had revealed that PVCs can contribute to cardiomyopathy and heart failure and treating PVCs could lead to improved cardiac function. PVC-induced cardiomyopathy was a potentially reversible condition in which left ventricular dysfunction is induced by frequent PVCs and function improves on suppressing $\mathrm{PVCs}^{(3)}$. The concept of PVC-induced cardiomyopathy was first proposed by Duffee and co-workers ${ }^{(\mathbf{( 4 )}}$ who noticed a small group of patients with cardiomyopathy recover normal left ventricular (LV) function after pharmacological suppression of frequent $\mathrm{PVCs}$ (5) . However, traditional echocardiographic methods may not effectively evaluate subtle and early forms of PVC-induced ventricular function impairment. Conventional 2dimensional (2D) STE provided a new convenient and non-invasive method for quantitative evaluation of cardiac function by allowing to measure strain values ${ }^{(6)}$. Premature ventricular complex-induced cardiomyopathy 
is a potentially reversible condition in which left ventricular dysfunction is induced by the occurrence of frequent premature ventricular complexes (PVCs ) ${ }^{(7)}$.

\section{Aim of the work}

The aim of this work was to assess feasibility of using 2-dimensional speckletracking echocardiography (2D-STE) for early detection of subtle LV systolic dysfunction in patients with PVCs without structural heart disease, detection of risk factor and frequency of PVCs associated with PVCs induced cardiomyopathy which might be useful for early detection and risk stratification of cardiac function impairment and to consider PVC inhibiting treatment if appropriate.

\section{PATIENTS and METHODS}

The ethical approval was obtained from the hospital ethical research committee and each patient entering the study was signed an informed consent. Fifty patients included in this study with asymptomatic frequent PVCs. All were without structural heart disease. Detailed history, physical examination, full labs, resting ECG, Holter monitoring for 24 hours, conventional ECHO assessment and finally strain imaging with measurement of LV-GLS were done. They were recruited from Cardiovascular Department at AlAzhar University Hospitals during the period from December 2017 to November 2018. In this study we assessed speckle tracking of LV in apparently normal patients with frequent PVCs.

\section{Patient's selection}

A) Inclusion criteria in group A: the study included all patients with asymptomatic frequent PVCs without structural heart disease and normal conventional echocardiographic examination.

B) Exclusion criteria: the study excluded patients with impaired systolic function ( EF < $50 \%$, patients with a significant valvular heart disease , myocardial diseases and pericardial disease., congenital heart disease. history suggestive of ischemic heart disease, left ventricular hypertrophy, uncontrolled hypertensive patient, diabetic patient using insulin, patient with a history of atrial fibrillation or cardiomyopathies and patients with chronic systemic or inflammatory diseases and any form of malignancy were also excluded from this study.

Control group (Group B) 20 volunteers (22-60 years old) with similar demographic characteristics but without VESs were included as the control group.
Methods:

(1) Clinical evaluation: included full history taking, general and local examination.

(2) Resting 12-leads (ECG): was performed to detect rate, rhythm, morphology, origin of PVCs, evidence of ischemic heart disease, evidence of other arrhythmia.

(3) 24-hour Holter monitoring : All recordings will be reviewed and edited manually on cardiovision software package, that can communicate with MedTech devices for detection of Average, maximum and minimum heart rate, Confirmation of presence of frequent PVCs, Calculation of PVC burden, presence of interpolated PVCs, presence of runs of non-sustained ventricular tachycardia ( NSVT), Exclusion of presence of other tachyarrhythmia and identifiable grading of ventricular arrhythmia risk according to the Lown's grade ${ }^{(8)}$.

(4) Resting conventional Trans-Thoracic Echocardiography: Images were obtained using (Philips iE 33, Philips Affiniti 50) with patients in the left lateral position at end of expiration according to the recommendations of the American society of Echocardiography ${ }^{(9)}$., All standard measurements were obtained in the parasternal long- and short-axis views; apical 4-chamber, 2-chamber and apical long axis views including (left ventricular end-diastolic diameter(LVEDD), left ven- tricular end-systolic diameter(LVESD), interventricular septum thickness(IVST), and left ventricular posterior thickness were measured(LVPWT), left ventricular end-diastolic volume (LVEDV) and left ventricular end-systolic volume (LVESV) were measured, and left ventricular ejection fraction (LVEF) was calculated by biplane modified Simpson's method ${ }^{(10)}$.

(5) Speckle Tracking Echocardiography (STE): With simultaneous ECG recording, 2D echocardiography images were obtained from apical window (to get $\mathrm{AP} 4 \mathrm{C}, \mathrm{AP} 2 \mathrm{C}$ and $\mathrm{AP} 3 \mathrm{C}$ views), Longitudinal 2D speckle-tracking strain 
Assessment of the effects of frequent ventricular extra systoles on the left ventricle...

values were obtained from one representative cycle. We chose to analyze the cardiac cycle with the best tracking and visually most satisfactory strain curves. The tracking quality was visually assessed and corrections were made if necessary. SLS values were based on the American Society of Echocardiography's 17-segment LV model, Segmental LS was calculated as the percentage of lengthening or shortening of each segment and global longitudinal stain (GLS) was calculated as the average of regional strains. The results of all three planes were represented in a single bull's-eye summary as global peak longitudinal strain (GPLS) ${ }^{(11)}$

Statistical analysis: data were tabulated and analyzed using the computer program SPSS (Statistical package for social science) version 20. Quantitative data were expressed as mean standard deviation (SD). Qualitative data were expressed as frequency and parentage.

The following tests were done: independentsamples t-test of significance: was used when comparing between two means. Chi-square test was used when comparing between nonparametric data.

Probability (P-value) P-value $<0.05$ was considered significant. P-value $<0.001$ was considered highly significant. P-value $>0.05$ was considered insignificant. Receiver operating characteristics (ROC) curves: was used to detect cut-off point, sensitivity, specificity, positive predictive value (PPV) and negative predictive value (NPV). Diagnostic sensitivity: it measured the incidence of true positive results in patients group. Diagnostic specificity: it measured the incidence of true negative results in a non-diseased group(Control group). Positive predictive value: it was the percent of true positive results among all positive results. Negative predictive value: It was the percent of true negative results among all negative results.

\section{RESULTS}

There was no statistically significant difference between patients and control as regard age $(\mathrm{P}$-value $=0.13)$. The mean age of patients was $45.98 \pm 11.91$ years while that of controls was $41.25 \pm 11.70$ years.

There was no statistically significant difference between the patients and control as regard gender $(\mathrm{P}$-value $=0.35)$. Patients were $42 \%$ males and $58 \%$ females, while controls were $30 \%$ males and $70 \%$ females.

There was no statistically significant difference (P-value > 0.05) between the patients and control as regarding risk factors, patients were $30 \%$ hypertensive, $20 \%$ diabetic and $12 \%$ smoker, while control were $20 \%$ hypertensive, $20 \%$ diabetic and $15 \%$ smokers.

Table 1: comparison between the patients and control as regard 2D conventional echocardiography 2D speckle tracking echocardiography

\begin{tabular}{|l|l|l|l|}
\hline $\begin{array}{l}\text { Groups } \\
\text { Parameter }\end{array}$ & $\begin{array}{l}\text { Patients } \\
(\mathbf{n = 5 0 )}\end{array}$ & $\begin{array}{l}\text { Control } \\
(\mathbf{n = 2 0})\end{array}$ & p-value \\
\hline LVEDV & $118.54 \pm 23.63$ & $112.40 \pm 19.74$ & 0.30 \\
\hline LVESV & $53.16 \pm 16.14$ & $51.00 \pm 8.30$ & 0.57 \\
\hline EF & $59.46 \pm 4.94$ & $62.00 \pm 6.03$ & 0.07 \\
\hline AP3 & $-15.36 \pm 3.48$ & $-20.40 \pm 1.72$ & 0.001 \\
\hline AP4 & $-19.34 \pm 3.58$ & $-21.30 \pm 2.08$ & 0.025 \\
\hline AP2 & -17.243 .402 & -20.451 .669 & 0.001 \\
\hline GLS & $-17.20 \pm 2.79$ & $-20.85 \pm 1.49$ & 0.0001 \\
\hline
\end{tabular}

Table 1 showed no statistically significant difference $(\mathrm{P}$-value $>0.05)$ between patients and control as regard conventional echocardiography parameter, while there was a statistically significant difference regarding speckle tracking echocardiography between case and control groups ( $\mathrm{P}$-value $<0.05$ )

Table 2: correlation between global longitudinal strain of LV and PVCs burden

\begin{tabular}{|l|l|l|l|}
\hline & Variable & r & p-value \\
\hline
\end{tabular}




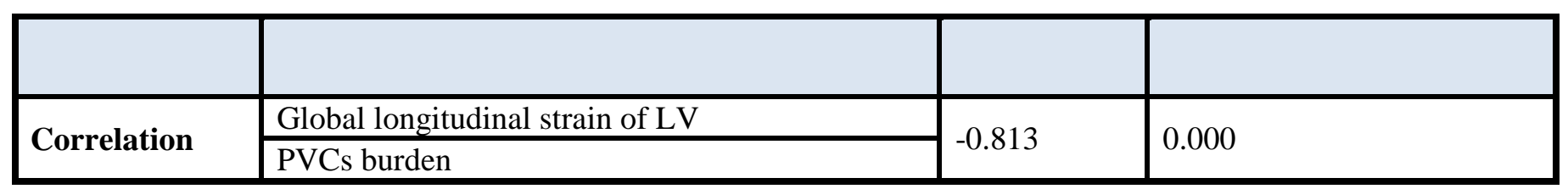

Table 2 showed statistically significant difference $(\mathrm{P}$-value $<0.05)$ between global longitudinal strain of LV and PVCs burden with negative correlation between both $(\mathrm{r}=-0.813)$

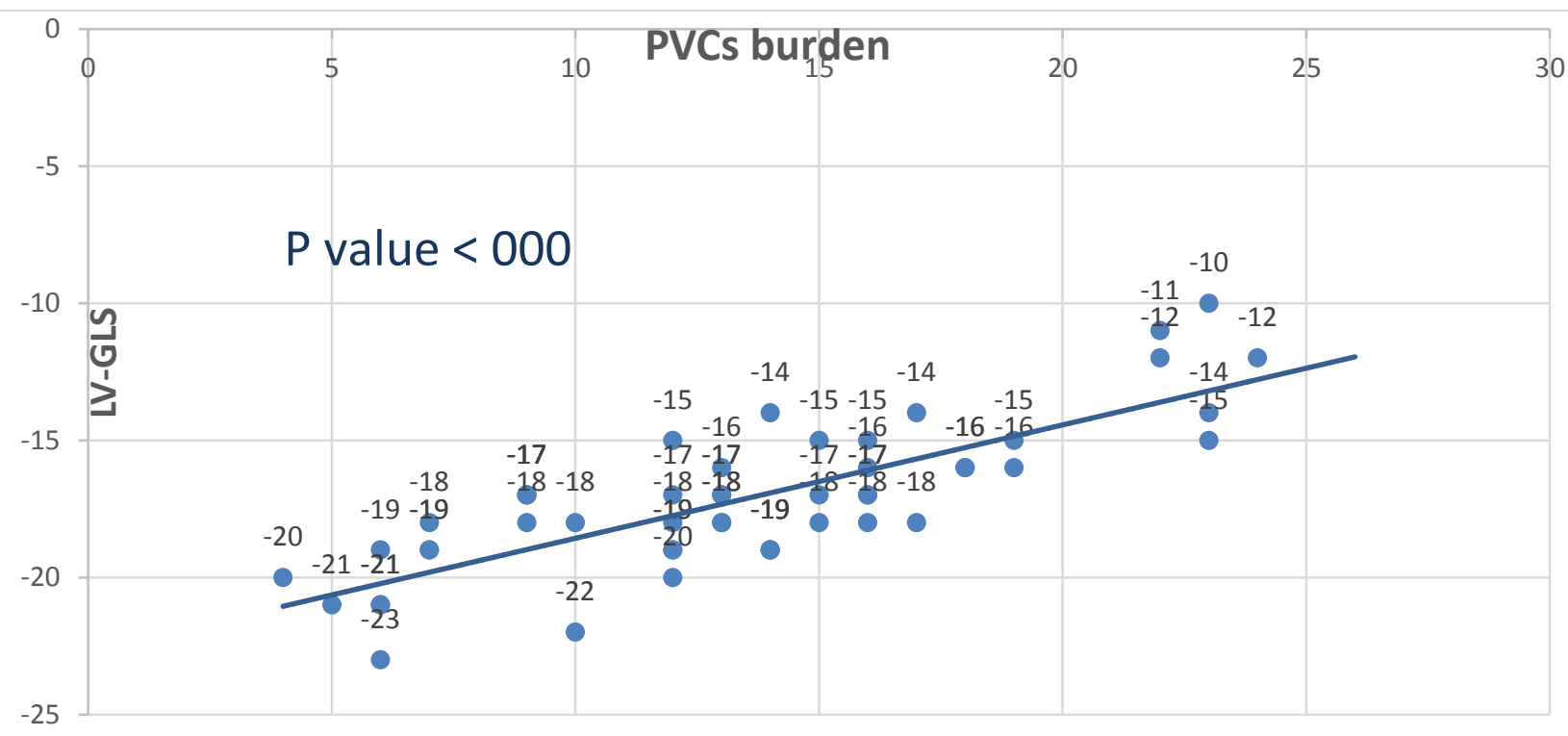

Fig.1: Correlation between global longitudinal strain of LV and PVCs burden.

Table 3: Correlation between global longitudinal strain of $L V$ and duration of PVCs complex

\begin{tabular}{|l|l|l|l|}
\hline \multirow{2}{*}{ Correlation } & Variable & $\mathbf{r}$ & p-value \\
\hline \multirow{2}{*}{ Global longitudinal strain of LV } & -0.616 & 0.001 \\
\cline { 2 - 5 } & Duration of PVCs complex & & \\
\hline
\end{tabular}

Table 3 showed statistically significant difference $(\mathrm{P}$-value $<0.05)$ between global longitudinal strain of LV and duration of PVCs complex with negative correlation between both $(r=-0.616)$.

Table 4: comparison between patient with and without risk factors associated with PVCs regarding $L V$-GLS

\begin{tabular}{|c|c|c|c|c|}
\hline Risk factors & Variable & LV-GLS & $\mathbf{r}$ & p-value \\
\hline \multirow{2}{*}{$\begin{array}{l}\text { Presence of } \\
\text { interpolated PVCs }\end{array}$} & With interpolated & $\begin{array}{ll}-17.74 & 2.418 \\
\end{array}$ & \multirow{2}{*}{-0.873} & \multirow{2}{*}{0.004} \\
\hline & Without interpolated & $\begin{array}{ll}-15.57 & 2.793\end{array}$ & & \\
\hline \multirow{2}{*}{ Focality } & Unifocal & $\begin{array}{ll}-17.89 & 2.596\end{array}$ & \multirow{2}{*}{-0.61} & \multirow{2}{*}{0.001} \\
\hline & Multifocal PVCs & $-1500 \quad 2258$ & & \\
\hline
\end{tabular}


Table 4 showed statistically significant difference (P-value < 0.05) between LV-GLS with interpolated and multifocal PVCs with negative correlation between both $(\mathrm{r}=-0.873)(\mathrm{r}$ $=-0.61)$ respectively.

\section{Discussion}

In this study, our aim was to evaluate LV systolic function in patients with PVCs without structural heart disease and early detection of subtle dysfunction by using 2dimensional speckle-tracking echocardiography (2D-STE), detection of risk factors and frequency of PVCs associated with PVCs induced cardiomyopathy which might be useful for early detection and risk stratification of cardiac function impairment and to consider PVC inhibiting treatment if appropriate.

In the present study, 50 consecutive patients aged 23 - 65 years, mean $45.96 \pm 11.88 ; 58 \%$ of the studied patients were females and $42 \%$ of them were male with asymptomatic PVCs were included and their ECHO findings were compared to those of 20 apparently healthy age and sex-matched individuals. Regarding 2D conventional echocardiographic parameters, there was no significant difference between the case and control groups, in case group mean LV-EF was $59.4600 \pm 4.945$ and in the control group $62.0000 \pm 6.03499$ with no $(\mathrm{P}=0.07)$. LV- EDV in case group mean was $123 \pm 35$ while, in the control group was $117 \pm 29$, it seemed to be mild increased in case group, but without statistically significance $(\mathrm{P}=0.391)$. These findings are in agreement with another studies $(5,12)$ who found no statistically difference between the PVCs group (49) and control group (20) regarding conventional 2D echocardiography. As regard 2D speckle tracking variables of global function, in our study there was a significant difference regarding LV-GLS between case and control groups. Global values of LV were significantly lower in the PVCs group than in the control subjects with $\mathrm{P}<0.001$. This indicates that PVCs may induce early LV dysfunction not detected by conventional 2D echocardiography measurements and this finding coincides with those of Wijnmaalen et al. ${ }^{(13)}$ and Lie et al. (14), as regard PVCs burden, all of case group patients had PVCs with total number of PVCs (PVCs burden) ranging between $4 \%-24 \%$. There was a significant inverse correlation between PVCs burden and LV-EF, LV-GLS. The correlation was moderate for LV-EF \& very strong for LV-GLS. These finding coincides with those of Del Carpio et al. ${ }^{(15)}$ which regarded correlation between PVCs burden and LV function. They showed that the frequency of PVCs correlates at least modestly with the extent of LV dysfunction. However, there are no clear-cut points that mark the frequency at which cardiomyopathy is unavoidable

A cut-off point of $24 \%$ had been proposed as having the best sensitivity and specificity for the prediction of cardiomyopathy with a sensitivity of $79 \%$ and specificity of 78.5\% However, we cannot currently consider a clear cut-off value, as PVCs induced cardiomyopathy can be observed even in patients with lower PVC burden $(>10 \%)^{(1)(2)}$, while Lie et al. ${ }^{(14)}$ concluded that more than $8 \%$ PVC was associated with impaired myocardial function by GLS, which was a considerably lower threshold of \%PVC than previously reported. This finding indicates that patients with more than approximately 8000 PVCs/24 h should undergo strain echocardiography to evaluate cardiac function and to consider PVC inhibiting treatment if appropriate. In our study By ROC analyses, a $\%$ PVC $>12.5$ optimally detected patients with a GLS worse than $-18 \%$, with a sensitivity of $80 \%$ and specificity of $67 \%$ and an area under the curve (AUC) of 0.79 . There was a strong significant correlation between higher \%PVC and worse GLS $(\mathrm{R}=0.813, \mathrm{P}=0.001)$ while, there was a moderate significant correlation between \%PVC and EF $(\mathrm{R}=0.491, \mathrm{P}=0.01)$. Therefore, although burden was significant, it was not the only factor contributing to impairment of LV systolic function (16). According to PVCs origin patients with PVCs were classified into 4 subgroups. No significant differences were seen in LV-EF, LV-EDV and LV-ESV between the 4 groups whereas PVCs originating from the RVOT were associated with a dysfunction by decrease strain in speckle tracking in comparison to other groups at the same burden, These findings are similar to findings of Del Carpio ${ }^{(14)}$ who found that right ventricular (RV) PVCs could cause LVEF impairment at a lower daily burden than those originating in the LV (10\% versus $20 \%$ burden, respectively), in our study by using subgroups analysis a cut off value of a PVC burden $\geq 11 \%$ were associated with a dysfunction by decrease strain in speckle tracking in patients with RVOT origin, whereas 
PVCs originating from the LVOT were associated with dysfunction by speckle tracking only at a PVC burden $\geq 18 \%$.

Regarding PVCs focality when we compared patients with multifocal versus patients with unifocal PVCs regarding strain imaging parameters included in our study we found a more decrease in LV longitudinal strain $(-15.82 \pm 1.75 \mathrm{Vs}-17.64 \pm 2.93)(\mathrm{P}$ $=9909$ i98 0.05) in patients with multifocal PVCs than of patients with unifocal PVCs and theses differences were statistically significant and these finding coincides with those Wijnmaalen et $_{\text {al }} .^{(13)}$ and Del Carpio ${ }^{(15)}$ study when they compared patients with reduced LVEF with those with normal LVEF found that patients with reduced LVEF were more likely to have multiform $\mathrm{PVCs}^{(2)}$. Regarding QRS Features and Interpolation, in our study we compared 14 patients with interpolated PVCs with 36 patients without interpolated PVCs. Patients with interpolated PVCs had slightly lower LV-EF in comparison to patients interpolated PVCs $(57.14 \pm 3.15$ Vs $60.36 \pm$ 5.24, $\mathrm{P}=0.312$ ) but this difference was statistically insignificant. While there was a statistically significant decrease in global LV strain values than patients without interpolated PVCs $(-17.86 \pm 2.57 \mathrm{Vs}-15.64 \pm 2.67)(\mathrm{p}=$ 0.01 ) and these finding coincides with those of Olgun et al. ${ }^{(17)}$. As regard QRS features we noticed week correlation between duration of complex and LV-EF with statistically significant correlation $\mathrm{p}=0.01$ and there was strong correlation between duration of complex and LV-EF with statistically significant correlation $p<0.001$ and these finding is in agreement with those of Yokokawa et al. ${ }^{(18) .}$

\section{Conclusion}

This study concluded that premature ventricular complexes may produce $\mathrm{LV}$ dysfunction and cardiomyopathy independently of any pre-existing underlying cardiac disease and early detection of subtle LV dysfunction can be done by speckle tracking echocardiographic modalities other than conventional echocardiographic measures in asymptomatic PVCs patient.

\section{References}

1. Winkens A, Hoppener F, Kragten A, Verburg P and Crebolder F (2014): Are premature ventricular contractions always harmless? . Eur. J. Gen. Pract., 20:134-138.
2. Lin Y, Chang L, Lin J, Lo W, Chung P, Chen Y and Chao F (2015): Longterm outcome of multiform premature ventricular complexes in structurally normal heart .Int. J. Cardiol., 180: 8085.

3. Krynski T, Stec S, Szmit S, Zaborska B, Balsam P, Kulakowski $P$ and Opolski G (2014): Impact of radiofrequency catheter ablation onechocardiographic and cardiopulmonary performance in patients with ventricular extrasystolic beats and suspected arrhythmiainduced cardiomyopathy. Heart Vessels, 29: 808-816.

4. Duffee $F$, Shen $K$ and Smith $H$ (1998): Suppression of frequent premature ventricular contractions and improvement of left ventricular function in patients with presumed idiopathic dilated cardiomyopathy . Mayo Clin. Proc., 73: 430-433.

5. Lee $A$ and Deyell $M$ (2016): Premature ventricular contractioninduced cardiomyopathy. Current Opinion in Cardiology, 31(1): 1-10.

6. Ling $Y$, Wan $Q$, Chen $Q$ and Zhu W (2017): Assessment of subtle cardiac dysfunction in patients with frequent premature ventricular complexes by real-time three-dimensional speckle tracking echocardiography. Clinical Cardiology, 40(8): 554-558.

7. Yarlagadda K et al. (2005): Reversal of cardiomyopathy in patients with repetitive monomorphic ventricular ectopy originating from the right ventricular outflow tract.Circulation, 112: 1092-1097.

8. Lown B, Fakhro M, Hood B and Thorn W (1969): The coronary care unit. New perspectives and directions. Jam. Med. Assoc., 199:188-198.

9. Lang $A$ and Roberto $M$ (2015): Recommendations for cardiac chamber quantification by echocardiography in adults: an update from the American Society of Echocardiography and the European Association of Cardiovascular Imaging. Journal of the American Society of Echocardiography, 28(1): 1-39

10. Nagueh S F and Han F (2016): Recommendations for the evaluation of 
left ventricular diastolic function by echocardiography: an update from the American Society of Echocardiography and the European Association of Cardiovascular Imaging. European Journal of Echocardiography, 17(12): 13211360.

11. Gorcsan J and Tanaka H (2014): Echocardiographic assessment of myocardial strain. Journal of the American College of Cardiology, 58(14): 1401-1413.

12. Park Y et al. (2014): Frequent Pemature ventricular complex is associated with left atrial enlargement in patients with normal left ventricular ejection fraction. Pacing and Clinical Electrophysiology, 37(11): 14551461.

13. Wijnmaalen AP, Delgado V, Schalij MJ, van Huls van Taxis CF, Holman ER, Bax JJ and Zeppenfeld $K$ (2010): Beneficial effects of catheter ablation on left ventricular and right ventricular function in patients with frequent premature ventricular contractions and preserved ejection fraction. Heart, 96 (16): 1275-1280.
14. Lie $\emptyset \mathrm{H}$ et al. (2017): Lower than expected burden of premature ventricular contractions impairs myocardial function. ESC Heart Failure, 4(4): 585-594.

15. Del Carpio $M \quad F$ et al.(2011): Characteristics of premature ventricular complexes as correlates of reduced left ventricular systolic function: Study of the burden, duration, coupling interval, morphology and site of origin of PVCs. Journal of Cardiovascular Electrophysiology, 22(7): 791-798.

16. Cha Y M et al. (2012): Premature ventricular contraction-induced cardiomyopathy: A treatable condition. Circulation: Arrhythmia and Electrophysiology, 5(1): 229-236.

17. Olgun H, Yokokawa M, Baman T, Kim HM, Armstrong W, Good E and Chugh $\mathbf{A}(\mathbf{2 0 1 1})$ : The role of interpolation in PVC-induced cardiomyopathy. Heart Rhythm, 8(9): 1046-1050.

18. Yokokawa M, Kim HM, Good E, Crawford T, Chugh A, Pelosi F $\mathrm{Jr}$, Jongnarangsin $\mathrm{K}$, Latchamsetty $R$ and Armstrong W(2012): Impact of QRS duration of frequent premature ventricular complexes on the development of cardiomyopathy. Heart Rhythm, 9(4):1460-1466. 